\title{
ChemComm
}

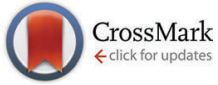

Cite this: Chem. Commun., 2015, 51, 17257

Received 20th August 2015, Accepted 29th September 2015

DOI: $10.1039 / \mathrm{c} 5 \mathrm{cc} 07012 \mathrm{k}$

www.rsc.org/chemcomm

\section{Aggregation-induced emission fluorogens as biomarkers to assess the viability of microalgae in aquatic ecosystems $\dagger$}

\author{
Feng Guo, ${ }^{\text {abc }}$ Wei-Ping Gai, ${ }^{c}$ Yuning Hong, ${ }^{d}$ Ben Zhong Tang, ${ }^{e}$ Jianguang Qin*a \\ and Youhong Tang*b
}

\begin{abstract}
Microalgae can be a valuable indicator for monitoring water pollution due to their sensitivity to the changes induced by pollutants in the environment. In this study, an aggregation-induced emission fluorogen was used as a novel tool to differentiate dead and live microalgae and quantify the link between live algal concentration and fluorogen intensity. Protein in the cell protoplasm is the key component contributing to fluorescence emission in algae.
\end{abstract}

For the past few decades the oceans have been regarded as a giant dumping area for all types of disposal, from organic carbon, plastic, toxins and heavy metals to radioactive waste with the misconception that the gigantic size of the oceans is sufficient to dilute pollutants and render harmless any materials dumped in. ${ }^{1}$ In fact, the ocean ecosystem is currently under enormous stress from a variety of pollution sources. The oceans have a considerable self-purification function but that ability is not infinite. By dumping excessive pollutants into the oceans, humans may permanently alter the ocean ecosystem. Moreover, a number of manufactured chemicals can threaten human health, causing cancer, immune deficiency, nerve disorders and low fertility.

\footnotetext{
${ }^{a}$ School of Biological Sciences, Flinders University, Adelaide 5042, Australia. E-mail: jian.qin@flinders.edu.au

${ }^{b}$ Centre for NanoScale Science and Technology, School of Computer Science, Engineering and Mathematics, Flinders University, Adelaide 5042, Australia. E-mail: youhong.tang@flinders.edu.au

${ }^{c}$ Department of Surgery, Centre for Neuroscience, School of Medicine,

Flinders University, Adelaide 5042, Australia

${ }^{d}$ School of Chemistry, The University of Melbourne, Melbourne 3010, Australia

${ }^{e}$ Department of Chemistry, The Hong Kong University of Science and Technology, Kowloon, Hong Kong, China

$\dagger$ Electronic supplementary information (ESI) available: Materials, algae strain and culture conditions, BSPOTPE monitoring live and dead N. oculata, monitoring PL intensity of live and dead $N$. oculata according to different time profiles, measurement of $N$. oculata staining by BSPOTPE under the control of salinity, evaluating the PL intensity of BSPOTPE with different densities of $N$. oculata in PBS buffer, live and dead $N$. oculata staining with BSPOTPE or propidium iodine and elucidation of the staining mechanism of $N$. oculata by BSPOTPE. See DOI: $10.1039 / \mathrm{c} 5 \mathrm{cc} 07012 \mathrm{k}$
}

Biological monitoring uses biological responses to evaluate changes in the environment with the intention of establishing a qualitative control program. Through systematic and regular monitoring, the responses of organisms are used to assess the impact of pollutants on the health and function of an aquatic ecosystem. Microalgae are vitally important to the food web in the aquatic ecosystem and can be a valuable indicator for monitoring water pollution due to their sensitivity to chemical changes in the environment. Moreover, microalgae are pivotal in the biogeochemical cycling of nutrients and pollutants in the ocean. ${ }^{2}$ Microalgae have been referred to as a "green liver" of the ocean, acting as an important sink for chemical compounds. Nannochloropsis is a genus of small green microalgae and is well known for its nutritional value and ability to produce valuable lipophilic and lipophobic materials. In the aquaculture industry, Nannochloropsis is extensively used as live feed to grow small zooplanktons such as rotifers and copepods in fish hatcheries. Nannochloropsis oculata ( $N$. oculata) is one of the six species in this genus. Nannochloropsis is a unicellular green alga having a spherical shape and $2-5 \mu \mathrm{m}$ in diameter. In this study, this ubiquitous species was used to test whether a new and unique luminogen can be a biomarker to assess the viability of microalgae.

Various species of microalgae have been used for the bioassessment of pollution in water. ${ }^{3}$ Recently, some methods have been developed to assess phytoplankton viability in marine and coastal environments, such as SYTOX green,${ }^{4,5}$ fluorescein diacetate (FDA) $)^{6-9}$ FDA + 5-chloromethylfluorescein diacetate $(\mathrm{CMFDA})^{10}$ and neutral red. ${ }^{11,12}$ Among these assays, fluorescein formed by intracellular hydrolysis of FDA leaks rapidly from cells ${ }^{13}$ and SYTOX green does not reveal the mechanism causing cell death. Auto-fluorescence in chlorophyll pigments overlaps with the fluorescence of propidium iodide (PI) ${ }^{14}$ whereas neutral red is not effective for staining some phytoplankton species due to leaching or cell shrinkage. ${ }^{15,16}$ So far, no efficient staining method has been found to easily differentiate live from dead algae. ${ }^{17}$

We previously discovered a group of unique luminogens which are nonluminescent when molecularly dissolved but highly fluorescent when aggregated. ${ }^{18}$ Aggregation-induced 
emission (AIE) describes this novel phenomenon and the restriction of intramolecular motions is the main cause of the AIE phenomenon. ${ }^{19}$ Over the past decade, AIE luminogens have been successfully employed in a variety of biological applications, ${ }^{19-21}$ such as long-term cell tracking, non-self-quenching DNA labelling, inhibition of amyloid fibrillation, differentiation of protein monomers, oligomers and fibrils, ${ }^{22}$ monitoring of cell apoptosis and long-term bacterial viability assays. ${ }^{23}$ AIE luminogens exhibit high quantum efficiency, good biocompatibility and appreciable photostability, and these features have motivated us to further explore their application in the thriving field of aquatic ecology.

Recently we discovered an AIE-active molecule, 1,2-bis[4-(3sulfonatopropoxyl)phenyl]-1,2-diphenylethene salt (BSPOTPE), for staining live particles. ${ }^{24}$ BSPOTPE is a water-soluble and biocompatible fluorogen without fluorescence in physiological buffers. In the current study, we discovered an important function of BSPOTPE as a fluorescent probe to quantitatively differentiate live and dead $N$. oculata in algal culture.

Dead $N$. oculata $\left(10 \times 10^{6} \mathrm{ml}^{-1}\right)$ was obtained after preservation in $75 \%$ ethanol for $30 \mathrm{~min}$, followed by BSPOTPE/PI staining for $20 \mathrm{~min}$. Our major finding was that BSPOTPE induced an increase of fluorescence ( $>300$-fold) at BSPOTPE $(200 \mu \mathrm{M})$ by binding with live $N$. oculata, whereas dead cells released minimum emission stained with BSPOTPE (Fig. 1A). To obtain stable emission, optimising the binding condition with an increment of dye concentration was conducted. The photoluminescence (PL) intensity of BSPOTPE-bound live $N$. oculata increased with the increase of BSPOTPE concentration (Fig. 1A) and reached a plateau at 200-500 $\mu \mathrm{M}$. No obvious change in PL intensity was observed in BSPOTPE-bound dead $N$. oculata. The $200 \mu \mathrm{M}$ BSPOTPE was chosen to further differentiate dead and live cells. Live $N$. oculata could be instantly stained by BSPOTPE after gentle mixing, which is much quicker than that by the neutral red staining method (1-2 h). ${ }^{12,15}$ Staining time with FDA usually takes $<10 \mathrm{~min}$, but not all organisms can be stained, and the fluorescence signal is not stable. ${ }^{7,25}$ Peperzak and Brussaard assessed the vitality of phytoplankton with six dyes by flow cytometry. Only Calcein-AM stained Nannochloropsis, but the signals were not strong. ${ }^{9}$ Flow cytometry has been used for particle quantification in aquatic ecosystems since the 1980s. Despite its high sensitivity and rapid quantification in cell densities, ${ }^{26}$ it is unlikely to differentiate dead algal cells from live algae.

The time profiles of PL intensity of BSPOTPE binding with live and dead $N$. oculata were compared. The PL intensity of BSPOTPE with live $N$. oculata was very high at the beginning and showed little variation over time (Fig. 1B). The combination of dead $N$. oculata with BSPOTPE binding led to very low but constant PL intensity. As BSPOTPE had high stability for staining live $N$. oculata for at least 7 days, BSPOTPE could provide a long-term viability assay for staining live $N$. oculata. Furthermore, the algal cell shape remained intact after verification with trypan blue staining. Salinity may affect the binding efficiency of BSPOTPE on live $N$. oculata. We controlled salinity by fixing a total of $500 \mu \mathrm{l}$ of $N$. oculata/seawater and $2 \mathrm{ml}$ of
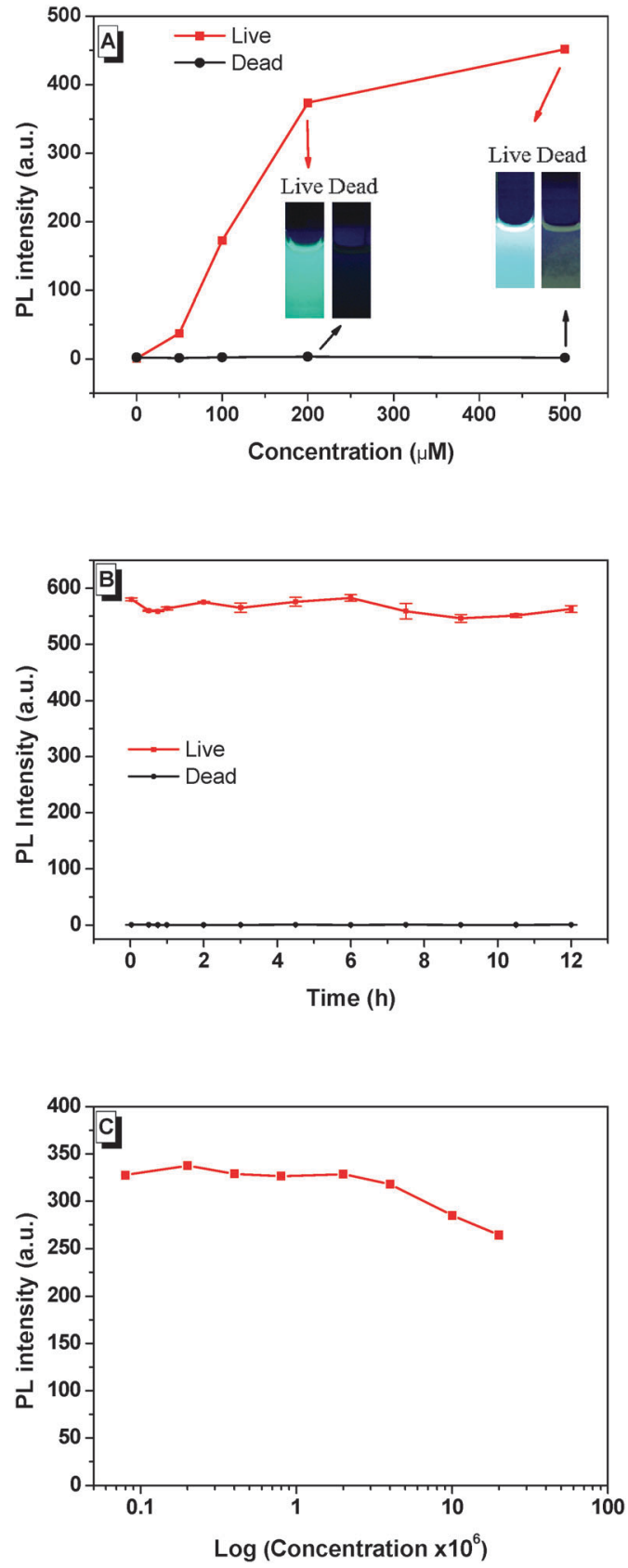

Fig. 1 Photoluminescence (PL) intensity of live and dead $N$. oculata at different BSPOTPE concentrations (A) and time elapse (B). The PL intensity of BSPOTPE at different $N$. oculata concentrations (C).

phosphate-buffered saline (PBS) at $13.8 \%$ salinity. The PL intensity was stable in a high concentration of live $N$. oculata (Fig. 1C). BSPOTPE could measure live algal concentration under light conditions regardless of salinity.

The binding of BSPOTPE to live cells can be visualised by fluorescence microscopy with strong blue fluorescence (Fig. 2B). The binding shows no overlap with the auto-fluorescence of chlorophyll pigments. On the other hand, the dead cells are almost non-emissive after binding to BSPOTPE (Fig. 2F). It is possible that $75 \%$ ethanol may denature the proteins on the cell membrane that could have been bound by BSPOTPE. In the 

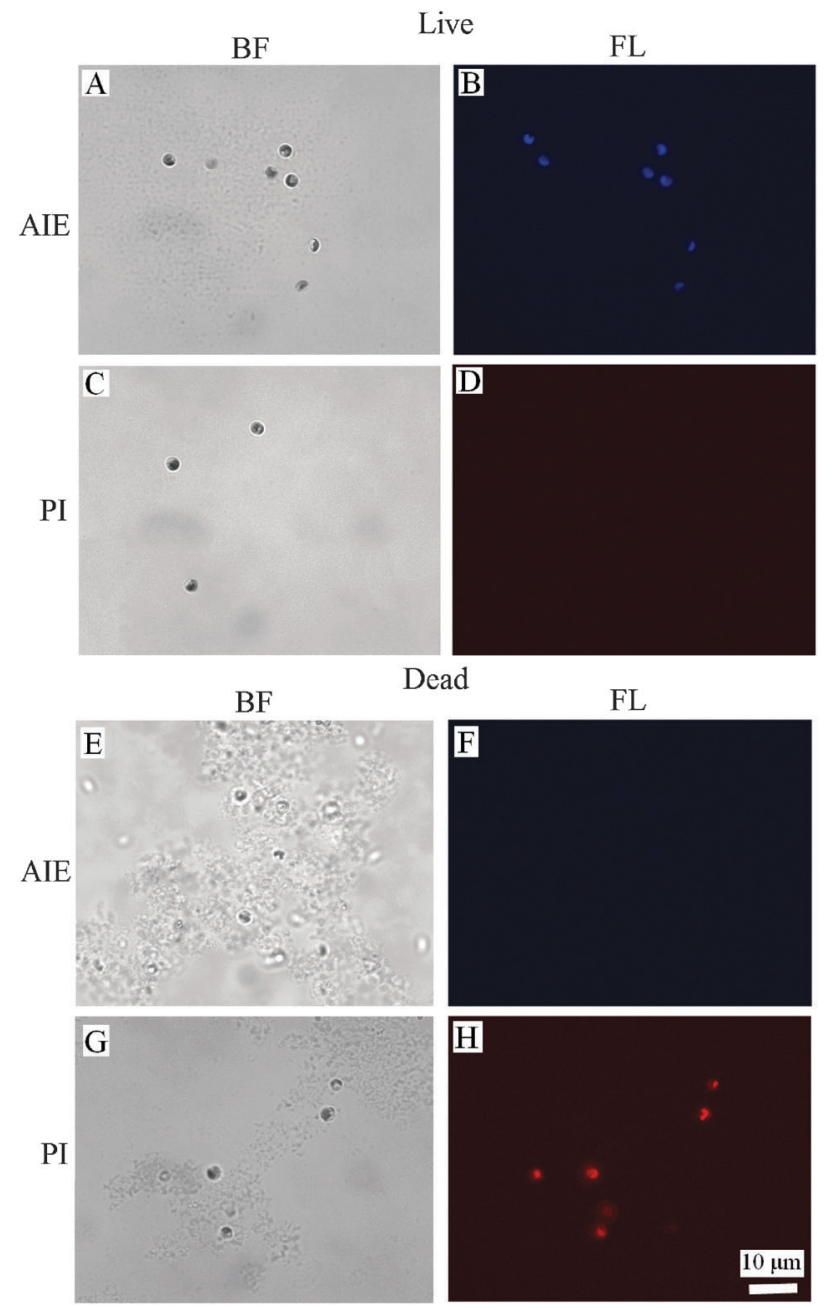

Fig. 2 ( $A, C, E$ and G) Bright-field (BF) and (B, D, F and H) fluorescence (FL) images of dead and live N. oculata stained with BSPOTPE (A, B, E and F) and PI (C, D, G and H) for $0.5 \mathrm{~h}$ in $200 \mu \mathrm{M}$ BSPOTPE.

present study, strong red fluorescence was achieved by the binding of PI and dead cells (Fig. 2H), though PI was nonemissive binding to live cells (Fig. 2D). It is well known that PI can stain dead cells as it is an impermeant nucleic acid dye that only stains cells with a damaged membrane, and the enhanced fluorescence can thus indicate the dead status of a cell. However, the method is not suitable for microalgae because of the overlap between auto-fluorescence of chlorophyll pigments and PI. ${ }^{14}$ PI is not capable of passing through the membranes of intact cells, and therefore should not be able to stain live cells, ${ }^{27,28}$ which explains why PI could not stain live $N$. oculata.

It is of interest to further explore the emission mechanisms in live $N$. oculata bound with BSPOTPE. The PL intensity was stable (within 300-350 a.u.) in a broad range of concentration of live N. oculata (Fig. 1C). Trypsin is a common enzyme that digests proteins into peptides and it only hydrolyses the peptide bonds in which the carbonyl group is contributed either by an arginine or lysine residue. BSPOTPE reduces fluorescence emission once it is combined with trypsin. The fluorescence was 157 a.u. prior to trypsin digestion and reduced to 128 a.u. after digestion by $1 \%$ trypsin for $2 \mathrm{~h}$ (Fig. 3A). Trypsin digestion reduced fluorescence by $18 \%$. In contrast to trypsin, proteinase $\mathrm{K}$ is a broad-spectrum serine protease. ${ }^{27}$ The predominant site of cleavage is the peptide bond adjacent to the carboxyl group of aliphatic and aromatic amino acids with blocked alpha amino groups. The fluorescence was 163 a.u. prior to proteinase $\mathrm{K}$ digestion and 28 a.u. after $0.5 \%$ proteinase digestion for $2 \mathrm{~h}$. Proteinase digestion reduced fluorescence by $83 \%$, suggesting that proteinase is stronger than trypsin in proteinlysis (Fig. 3B). Our data suggest that the fluorescence of live $N$. oculata may exist in the cell protoplasm. We can thus assume that protoplasm proteins inside the cell are the main binding sites of BSPOTPE, rather than the proteins on the cell surface. The staining mechanism may be similar to neutral red which can be accumulated in the cytoplasm and/or vacuoles in plant cells. ${ }^{15}$

The BSPOTPE solution in PBS emitted dim luminescence at $390 \mathrm{~nm}$ in the absence of $N$. oculata. When the density of $N$. oculata was low in PBS, the BSPOTPE solution became luminescent. The FL intensity at $485 \mathrm{~nm}$ kept rising with an increase in the $N$. oculata concentration diluted with PBS. The rate of FL enhancement kept almost constant up to $20 \times 10^{6}$ $N$. oculata per ml (Fig. 4). At a N. oculata concentration of $20 \times$ $10^{6} \mathrm{ml}^{-1}$, the $\mathrm{FL}$ intensity increased five-fold. The detection threshold reduced to $0.2 \times 10^{6}$ per $\mathrm{ml}$ of $N$. oculata. In the $N$. oculata range of $0.2-20 \times 10^{6} \mathrm{ml}^{-1}$, the plot of FL enhancement as a function of $N$. oculata concentration is linear with a high
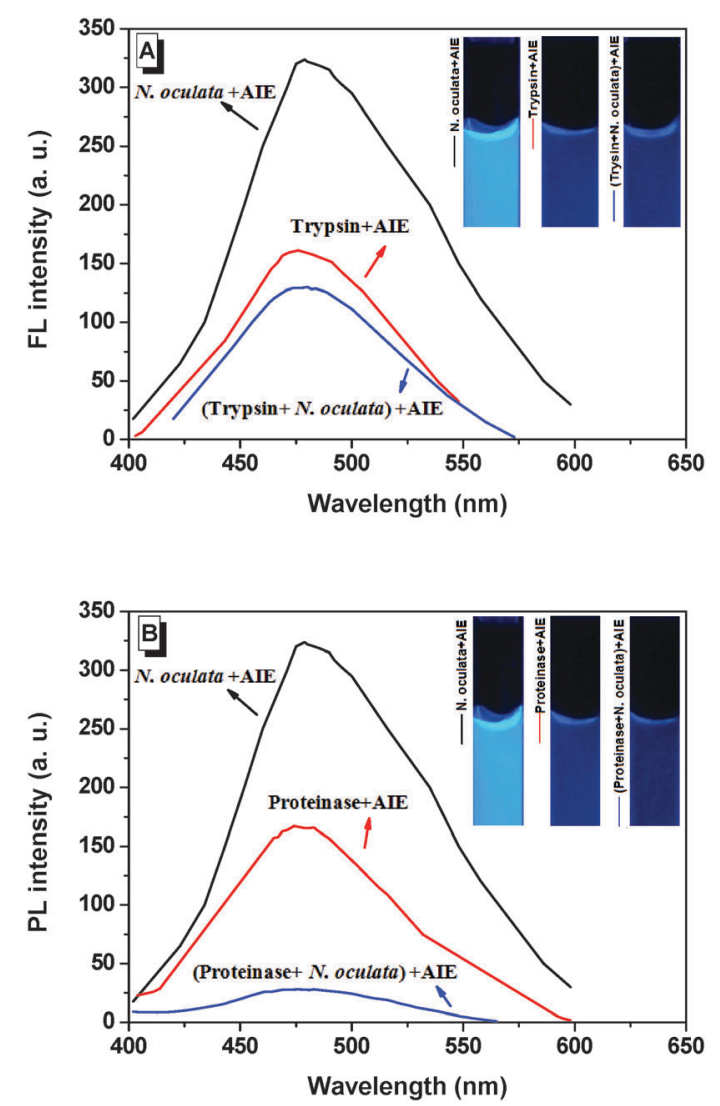

Fig. 3 Change of photoluminescence $(P L)$ intensity in live $N$. oculata before and after (A) trypsin or (B) proteinase $\mathrm{K}$ digestion. 


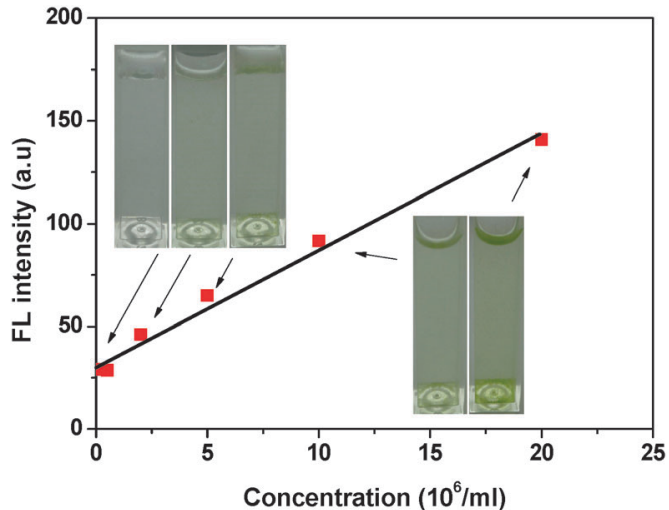

Fig. 4 Change in FL intensity at $475 \mathrm{~nm}$ at different $N$. oculata concentrations.

correlation coefficient $\left(r^{2}=0.989\right)$, indicating that the AIE luminogen can be used for a quantitative assay for $N$. oculata concentration in a PBS buffer.

Cell density and chlorophyll $a$ concentration are conventional measures for algal abundance but are insufficient to accurately reflect the physiological responses to ambient stress. ${ }^{29,30}$ For instance, algae may be deformed, decrease in cell size, or cease cell division in response to exposure to lethal metals or phytotoxic chemicals. ${ }^{29-31}$ Furthermore, chlorophyll $a$ concentrations may overestimate the viability of algae because of interference by other pigments (e.g. pheophytins). ${ }^{32,33}$ This study contributes to the understanding of the accuracy, precision and utility of the BSPOTPE as a viability measure. Compared with commercial viability probes, this dye is highly emissive, photostable and ease to use, making it suitable for long-term microalgae viability assays. To our best knowledge, this is the first study reported using an AIE fluorogen in microalgae. The great difference in fluorescence signals prompted us to conclude that BSPOTPE can be used for the $N$. oculata viability assay and may be employed as a biomarker for assessing the functional performance of other primary producers in aquatic ecosystems.

F.G., Y.H., W.G. and B.Z.T. thank NHMRC Early Career Fellowship (APP1037798), McKenzie Fellowship of the University of Melbourne, NHMRC Research Fellowship (535014) and Guangdong Innovative Research Team Program (201101C0105067115) for support.

\section{Notes and references}

1 Ocean disposal of radioactive waste: Status report. Dominique P. Calmet. IAEA Bulletin, 4, 1989.

2 C. A. Van Gestel and T. C. Van Brummelen, Ecotoxicology, 1996, $5,217$.

3 J. Ma, R. Zheng, L. Xu and S. Wang, Ecotoxicol. Environ. Saf., 2002, 52, 5.

4 L. C. Rai, J. P. Gaur and C. J. Soeder, Adv. Limnol., 1994, 42, 283.

5 M. J. W. Veldhuis, G. W. Kraay and K. R. Timmermans, Eur. J. Phycol., 2001, 36, 167.

6 A. C. Baudoux, M. J. W. Veldhuis, A. A. M. Noordeloos, G. van Noort and C. P. D. Brussaard, Aquat. Microb. Ecol., 2008, 52, 69.

7 J. D. Brookes, S. M. Geary, G. G. Ganf and M. D. Burch, Mar. Freshwater Res., 2000, 51, 817.

8 M. Garvey, B. Moriceau and U. Passow, Mar. Ecol.: Prog. Ser., 2007, 352, 17.

9 L. Peperzak and P. D. Brussaard, J. Phycol., 2011, 47, 692.

10 M. C. Villac and I. Kaczmzrska, Mar. Ecol.: Prog. Ser., 2011, 425, 47.

11 M. K. Steinberg, E. J. Lemieux and L. A. Drake, Mar. Biol., 2011, 158, 1431.

12 D. T. Elliott and K. W. Tang, Limnol. Oceanogr.: Methods, 2009, 7, 585.

13 E. M. Zetsche and F. J. R. Meysman, J. Plankton Res., 2012, 34, 493.

14 R. P. Haugland, J. Gregory and T. Z. Spence Michelle, Handbook of Fluorescent Probes and Research Products, Molecular Probes, Inc, OR, 9th edn, 2002, p. 605.

15 M. Sato, Y. Murata, M. Mizusawa, H. Iwahashi and S. Oka, Cult. Coll., 2004, 53.

16 R. W. Crippen and J. L. Perrier, Biotech. Histochem., 1974, 49, 97.

17 Z. Pavlic, Z. Vidakovic-Cifrek and D. Puntaric, Chemosphere, 2005, 61, 1061.

18 J. Luo, Z. Xie, J. W. Y. Lam, L. Cheng, B. Z. Tang, H. Chen, C. Qiu, H. S. Kwok, X. Zhan, Y. Liu and D. Zhu, Chem. Commun., 2001, 1740.

19 D. Ding, K. Li, B. Liu and B. Z. Tang, Acc. Chem. Res., 2013, 46, 2441.

20 J. Huang, N. Sun, J. Yang, R. Tang, Q. Li, D. Ma and Z. Li, Adv. Funct. Mater., 2014, 24, 7645.

21 J. Yang, N. Sun, J. Huang, Q. Li, Q. Peng, X. Tang, Y. Dong, D. Ma and Z. Li, J. Mater. Chem. C, 2015, 3, 2624.

22 C. W. T. Leung, F. Guo, Y. Hong, E. Zhao, R. T. Kwok, N. L. Leung, S. Chen, N. N. Vaikath, O. M. El-Agnaf, Y. Tang, W. P. Gai and B. Z. Tang, Chem. Commun., 2015, 51, 1866.

23 E. Zhao, Y. Hong, S. Chen, C. W. T. Leung, C. Y. Chan, R. T. Kwok, J. W. Lam and B. Z. Tang, Adv. Healthcare Mater., 2014, 3, 88.

24 F. Wang, J. Wen, L. Huang, J. Huang and J. Ouyang, Chem. Commun., 2012, 48, 7395.

25 A. Reynolds, G. Mackiernan and S. Van Valkenburg, Estuaries Coasts, 1978, 1, 192.

26 J. Dorsey, C. M. Yentsch, S. Mayo and C. McKenna, Cytometry, 1989, $10,622$.

27 C. M. Yentsch and S. A. Pomponi, Int. Rev. Cytol., 1986, 105, 183.

28 E. Kraus, H. H. Kiltz and U. F. Femfert, Hoppe-Seyler's Z. Physiol. Chem., 1976, 357, 233.

29 L. Shi, S. Günther, T. Hübschmann, L. Y. Wick, H. Harms and S. Müller, Cytometry, Part A, 2007, 71, 592.

30 N. Nyholm, Water Res., 1998, 19, 273.

31 M. Chao and C. Chen, J. Hazard. Mater., 2001, 82, 129.

32 D. Mares, A. Bonora, G. Sacchetti, M. Rubini and C. Romagnoli, Cell Biol. Int., 1997, 21, 397.

33 H. Rai, Arch. Hydrobiol., Beih. Ergeb. Limnol., 1980, 14, 3313. 